
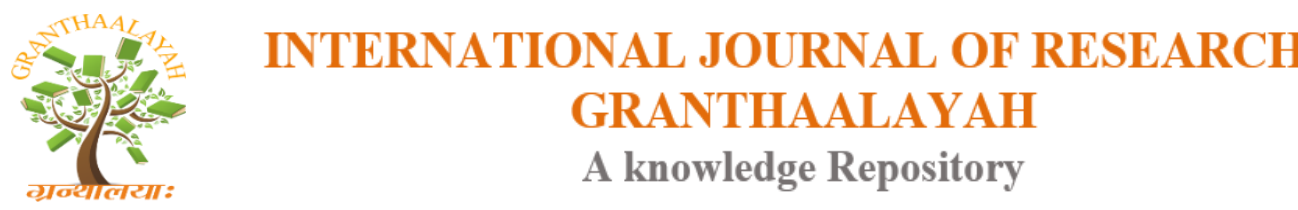

Science

\title{
AN AYURVEDIC MANAGEMENT OF SUBCLINICAL HYPOTHYROIDISM - A CASE REPORT
}

\author{
Dr. Dnyaneshwar Kantaram Jadhav *1 \\ ${ }^{* 1}$ M.D (Kaychikitsa) (Ayu), Assistance professor, Kaychikitsa department, Shri Dhanwantri \\ Ayurvedic Medical College \& Research Centre, India -281401
}

\begin{abstract}
Ayurved is science of life. Its guide human being for living on preventive \& curative both aspect. Its ancient knowledge which is still stand truth to time. There are many diseases which is not mentioned directly in Ayurveda texts is called as Anukta vikar. Subclinical hypothyroidism is one of such disease. Such disease specifically mentioned but its line of treatment described very well. The present case is 28 year old male patient was suffering from Bhaar vrudhi (weight gain) from last 2.5 years while symptoms like Drubalya (fatigue), Sheet prachiti (feeling excessive cold), Bhrama (vertigo), Shwasakashtata (dyspnoea after walking), Katishool (backache) since last 2 years.

Patient on modern medicine still he doesn't get any satisfy relief, at the end patient decide to take Ayurvedic treatment only. For treatment patient was came to Nakshatra Ayurved Panchkarma clinic \& Research center, Mumbai.

Ayurvedic management include internal medicine, Rukshaya bashpa peti sweda. After 1.5 month patient got Excellent Result. All symptoms disappear, weight reduces up-to $4 \mathrm{~kg}$, TSH level comes from 7.71 to 3.23 . This is single case study, will Collect data of more cases for further Research.

Keywords: Subclinical Hypothyroidism; Ayurvedic Management; Medovaha-Strotus; RasavahaSrotus; Dhatwagnimandya.

Cite This Article: Dr. Dnyaneshwar Kantaram Jadhav. (2018). "AN AYURVEDIC MANAGEMENT OF SUBCLINICAL HYPOTHYROIDISM - A CASE REPORT." International Journal of Research - Granthaalayah, 6(5), 481-486. https://doi.org/10.29121/granthaalayah.v6.i5.2018.1482.
\end{abstract}

\section{Introduction}

Nowadays most of patients came with other disease, but its supportive disease is hypothyroidism. Fast \& luxury lifestyle disturb doshas, which give birth to thyroid related disease.

Thyroid dysfunction is one of the most common endocrine imbalances seen in clinic. The two main clinical syndromes of the thyroid are over activity and under activity. Hypothyroidism is called as underactive thyroid, in which thyroid gland does not produced enough thyroid 
hormone. ${ }^{(1)}$ Infertility, weight problems, depression and chronic tiredness the most frequent complications of hypothyroidism.

Ayurveda has description of thyroid conditions in terms of swelling of the thyroid gland with hyperthyroidism ${ }^{(2)}$, but a clear description of hypothyroidism is absent. There are many diseases which is not mentioned directly in Ayurveda texts is called as Anukta vikar. ${ }^{(3)}$ Subclinical hypothyroid is one of such disease. Such disease specifically mentioned but its line of treatment described very well. Hypothyroidism in Ayurved can be consider as Anukta vikara and discussed as vata-kafaj dushti-janya vhadhi.

In an era when modern science is progressing with advent of advanced techniques for disease, diagnosis and treatment, there is still no easy solution to treat innumerable disease. Current available medicines are even not capable to give relief on many diseases. While Ayurved has solution on Anukta vikar also.

\section{A case report as follow -}

A 28 year old male patient came to us with chief compliant of ${ }^{(4)}$

1) Bhaar vrudhi (weight gain).

2) Drubalya (fatigue).

3) Sheet prachiti (feeling excessive cold).

4) Bhrama (vertigo)

5) Shwasakashtata (dyspnoea after walking)

6) Katishool (backache)

Patient had above complaints since 2.5 years.

No H/o Dm / HTN, Asthma

\section{History of Personal Illness}

The patient having history of hypothyroidism from last 2.5 years. He was under modern medicine (Tab.thyronorm $75 \mathrm{mcg}$ ). Even consumption of modern medicine, patient have been suffering from Bhaar vrudhi (weight gain), Katishool (backache), Sheet prachiti (feeling excessive cold), Drubalya (fatigue), Bhrama (vertigo), Shwasakashtata (dyspnoea after walking). From last two months all this symptoms increased. For better treatment patient took treatment from Homeopathy doctors but doesn't get relief in any symptoms, then he decide to take Ayurved treatment. For Ayurvedic treatment he came to our clinic - Nakshtra Ayurved Panchkarma Clinic \& Research Center, Mumbai.

\section{Personal History}

Occupation: Working as Police.

\section{Ashtavidha parikshana}

Nadi $($ pulse $)=98 /$ min. (kapha-vata)

Mala $($ stool $)=$ Malavshtmbha .

Mutra $($ urine $)=$ Normal.

Jeeva $($ tounge $)=$ Eshat saam .

Agni $=$ Kshudhamandya. 
Shabda $($ speech $)=$ Rukshya $($ Hoarse voice $)$.

Sparsha $($ skin $)=$ shita .

Druka $($ eyes $)=$ Normal.

Akruti = Madhyama.

Bala = Madhyama .

Raktadaaba (B.P) $=110 / 80 \mathrm{~mm} / \mathrm{Hg}$.

\section{Material and Method}

\section{Material}

Table 1: Showing material used for study

\begin{tabular}{|l|l|l|l|l|}
\hline SR.NO & DRAVYA & DOSE & DURATION & ANUPANA \\
\hline 1 & Medohar gugul & $250 \mathrm{mg}$ & 2 BID After food & Luck warm water \\
\hline 2 & Kanchanaar gugul & $125 \mathrm{mg}$ & 2 BID before food & Luck warm water \\
\hline 3 & Arogyavardhini vati & $500 \mathrm{mg}$ & 1 BID before food & Luck warm water \\
\hline 4 & Trifala gugul & $250 \mathrm{mg}$ & 1 BID After food & Luck warm water \\
\hline 5 & Laghumalini vasant vati & $125 \mathrm{mg}$ & 1 BID After food & Luck warm water \\
\hline 6 & Gandhrva haritaki & $500 \mathrm{mg}$ & 2 HS & Luck warm water \\
\hline 7 & Dashamul granules & $2 \mathrm{Gm}$ & At morning & Luck warm water \\
\hline
\end{tabular}

Table 2: showing panchkarma done in study

\begin{tabular}{|l|}
\hline PANCHKARMA \\
\hline Ruksha peti sweda \\
\hline
\end{tabular}

\section{Method}

Centre of study: Nakshtra Ayurved panchkarma clinic \& research center, Mumbai. Type of study: Simple random single case study.

\section{Observations and Result}

Table 3: showing daily treatment with prognosis

\begin{tabular}{|c|c|c|c|c|c|}
\hline DAYS & $\mathbf{1}^{\mathrm{ST}}$ & $2^{\mathrm{ND}}$ & $\mathbf{3}^{\mathrm{RD}}$ & $4^{\mathrm{TH}}$ & $5^{\mathrm{TH}}$ \\
\hline TREATMENT & $\begin{array}{l}\text { Medicine } \\
(1,2)\end{array}$ & $\begin{array}{l}\text { Medicine } \\
(1,2)\end{array}$ & $\begin{array}{l}\text { Medicine } \\
(1,2) \\
+ \\
\text { Ruksha peti } \\
\text { sweda. }\end{array}$ & $\begin{array}{l}\text { Medicine } \\
(1,2) \\
+ \\
\text { Ruksha peti } \\
\text { sweda }\end{array}$ & $\begin{array}{l}\text { Medicine } \\
(1,2)\end{array}$ \\
\hline \multicolumn{6}{|l|}{ Symptom } \\
\hline $\begin{array}{l}\text { Bhaar vrudhi (weight } \\
\text { gain). }\end{array}$ & 92kg & $90 \mathrm{~kg}$ & $89 \mathrm{~kg}$ & $87 \mathrm{~kg}$ & 86 \\
\hline Drubalya (fatigue). & +++ & ++ & + & 0 & $\mathbf{0}$ \\
\hline $\begin{array}{l}\text { Sheet prachiti } \\
\text { (feeling excessive cold) }\end{array}$ & ++++ & +++ & ++ & + & + \\
\hline
\end{tabular}




\begin{tabular}{|l|l|l|l|l|l|}
\hline Bhrama (vertigo) & + & + & 0 & 0 & 0 \\
\hline $\begin{array}{l}\text { Shwasakashtata } \\
\text { (dyspnoea after walking) }\end{array}$ & ++ & + & + & + & 0 \\
\hline Katishool(backache) & ++ & ++ & + & 0 & 0 \\
\hline
\end{tabular}

Table 4: Showing changes in thyroid reports

\begin{tabular}{|l|l|l|l|}
\hline \multicolumn{4}{|c|}{ THYRIOD PROFILE } \\
\hline & BEFORE (8/7/2017) & AFTER (28/8/2017) & NORMAL RANGE \\
\hline T3 & 91 & 93 & $60-200$ \\
\hline T4 & 6.3 & 7.2 & $4.5-12$ \\
\hline TSH & $\mathbf{7 . 7 1}$ & $\mathbf{3 . 2 3}$ & $\mathbf{0 . 3 - 5 . 5}$ \\
\hline
\end{tabular}

Clinical examination of the patients revealed Regression of symptoms due to our Ayurvedic management. The patient had started improving symptoms within 7 days. After 1.5 months treatment patient cured subjective as well as objective.

\section{Discussion}

\section{Hetu of subclinical-hypothyriodism as -}

1) Ahar

- Improper and irregular diet

- Ambuja anup mamsa Matsya sevan (daily )

- Dadhi

2) Vihar

- Prolong standing

- Excessive running

- Ratri jagran

3) Manasika nidan- Chinta, bhaya, and vegavrodha causes vata vrudhi

\section{Sanprapti Ghatak}

Dosh: vata-kapha.

Dushya: Rasa, Rakta, meda

\section{Vikalpa Samprapti ${ }^{(5)}$}

Vata dosha- sheeta guna

Kapha dosha- manda, sheeta, guru

\section{Samprapti ${ }^{(6)}$}

Hypothyroidism is under activities Agni. Due to various hetu dhatu level Agni is inhibited. In this case we found Rasa-Medovaha srotus dushti hetu. This hetu cause vata and kapha prokop. Which leads Rasavaha and Medovaha srotus dushi.

- Due to Agnimandya of Medodhatu, virkut medo dhatu produced. Its cannot participate into asthi dhatu poshana. This vikrut medo dhatu accumulate in body which cause Bhaar vrudhi (weight gain). Bhaar vrudhi also sign of Rasavahadushti \& kapha vrudhhi in body.

- Katishool due to lack of asthi dhatu poshana \& vata vrudhi. 
- Sheet prachiti due to kapha \& vata Dosha (as vata is yogvahi its increased sheet guna double in strength).

- Drubalya (fatigue) due to vata vrudhi and rasa dushti.

- Bhrama (vertigo) due to vata vrudhi.

- Shwasakashtata (dyspnoea after walking) due to vata vrudhi.

Diagnosis of hypothyroidism with its symptoms along with blood test measuring T3, T4, TSH levels.

Table 5: showing how to Diagnosis of hypothyroidism with T3,T4,TSH levels.

\begin{tabular}{|l|l|l|}
\hline TSH & T4 & INTERPRETATION \\
\hline Normal & Normal & Normal thyroid function \\
\hline Elevated & Low & Over-hypothyroidism \\
\hline Normal & Low & central-hypothyroidism \\
\hline Elevated & Normal & subclinical-hypothyroidism \\
\hline
\end{tabular}

\section{Action of Drug and other Procedure in Management of Subclinical- Hypothyriodism}

Table 6: Showing action of drug \& other procedure done in study

\begin{tabular}{|c|c|c|}
\hline SR.NO & DRAVYA & ACTION \\
\hline 1 & Medohar gugul(7) & Medaghna, lekhan karya. \\
\hline 2 & Kanchanaar gugul ${ }^{(\mathbf{8})}$ & Kaphaghan \\
\hline 3 & Arogyavardhini vati ${ }^{(\mathbf{9})}$ & $\begin{array}{l}\text { Dhatwagnivardhana, malashodhaka,Pakwashyadushti } \\
\text { nashka. }\end{array}$ \\
\hline 4 & Trifala gugul ${ }^{(\mathbf{1 0})}$ & Rasayana, malavashbhanashak,lekshan. \\
\hline 5 & $\begin{array}{l}\text { Laghumalini vasant } \\
\text { vati }^{(\mathbf{1 1})}\end{array}$ & Rasayana mainly Work on rasa-rakta dhatu, shothahara. \\
\hline 6 & Gandhrva haritaki $^{(\mathbf{1 2})}$ & Anulomna, vatashulnashak \\
\hline 7 & Dashamul granules ${ }^{\mathbf{( 1 3 )}}$ & Vatashamak, dipan, shothaghna. \\
\hline 8 & Ruksha peti swedan & Vatashamna, kaphashman. \\
\hline
\end{tabular}

\section{Conclusion}

Due to change in modern life style Hypothyroidism cases increased day by days. Ayurved has best solution in such type of Anukta vhyadhi. With doshas vichar, Ayurved cure such disease \& act as apunarbhava also. This is one example of successful case of subclinical hypothyroid among my all cured thyroid case. I will do further research on same disease.

\section{References}

[1] Dr.harsh mohan, Textbook of pathology ( $5^{\text {th }}$ ed $)$, chapter-27, endocrine system, jaypee publication, New delhi.repeint-2006, page no-827.

[2] Dr.Brhamananda Tripathi, Charak Samhita of maharshi charak, Charakacnahdrika commentatory, Charaka samhita, part-1, 5th ed. Varanasi: Chaukambha Sanskrit sansthana; Reprint: 2016.sutra sthana, trishothiya Adhyaya 18/21; page no- 372 . 
[3] Dr.Brhamananda Tripathi, Charak Samhita of maharshi charak, Charakacnahdrika commentatory, Charaka samhita, part-1, 5th ed. Varanasi: Chaukambha Sanskrit sansthana; Reprint: 2016.sutra sthana, trishothiya Adhyaya 18/44-47; page no- 378.

[4] Sahu Dustidev Et \& All: "Hypothyroidism" An Ayurvedic Perspective - A Critical Review, IAMJ: Volume 3; Issue 1; January - 2015: page no-152.

[5] Vd.Gopakumar, Samsidhi- Gk's book on classical thoughts and clinical targets, hypothyroidism, $4^{\text {th }}$ edition, published by mylandoor ayurvedic research center, kerala, page no-108-115.

[6] Vd.manoj jagtap \& vd.priyanka mestri, a case report of subclinical hypothyroidism \& its ayurvedic management, Ayurved patrika : Published on December -2017 : vol -05,page no-46,47.

[7] Shri Krishna gopal,ras tantra saar va sidhprayoasangrha,krishnagopal ayurved bhavan,rajasthan ; part $-2,13^{\text {th }}$ edition, 2011,page no-190.

[8] Dr.Brhamananda Tripathi, Sharangdhar-samhita of pandit sarangadharacaya (with dipika hindi commentary), madhyama khanda; vataka kalpana, chapter 7. Verse no.99. Varanasi: Chukhambha prakashan, Reprint: 2010; page no.207.

[9] Gune GP Vd (2005) Aushodhigunadharmashtri, part-2, kalpa no-10. In: Gune GP Vd (Ed.), Reprint, India, page no- 208.

[10] Dr.Brhamananda Tripathi, Sharangdhar-samhita of pandit sarangadharacaya (with dipika hindi commentary), madhyama khanda; vataka kalpana, chapter 7. Verse no.83. Varanasi: Chukhambha prakashan, reprint: 2010; page no.206.

[11] Aushadhi Gundharma shatra by Vd. G. P. Gune, reprint -2005, part-4, kalpa no 78, page no-446.

[12] Gandharva haritaki (https://www.bimbima.com/ayurved/gandhavra-haritaki-churna-benifitsingredients-dosage/466/) : uploaded on 2 September 2014 \& downloaded on 2 may 2018

[13] Vd.Vishnu gogate, Dravyagunavidhyana,(1 $\left.1^{\text {st }} \mathrm{ed}\right)$, vaidyamitra publication,pune : 2008 ;page no168.

\footnotetext{
*Corresponding author.

E-mail address: dj85015@ gmail.com
} 